\title{
Impact of anharmonic effects on the phase stability, thermal transport, and electronic properties of AIN
}

\author{
Nina Shulumba, ${ }^{1}$ Zamaan Raza, ${ }^{1}$ Olle Hellman, ${ }^{1,2}$ Erik Janzén, ${ }^{1}$ Igor A. Abrikosov, ${ }^{1,3}$ and Magnus Odén ${ }^{1}$ \\ ${ }^{1}$ Department of Physics, Chemistry, and Biology (IFM), Linköping University, SE-581 83, Linköping, Sweden \\ ${ }^{2}$ Division of Engineering and Applied Science, California Institute of Technology, Pasadena, California 91125, USA \\ ${ }^{3}$ Materials Modeling and Development Laboratory, NUST “MISIS," 119049 Moscow, Russia
}

(Received 16 May 2016; published 26 September 2016)

\begin{abstract}
Wurtzite aluminium nitride (AlN) is a technologically important wide-band-gap semiconductor with an unusually high thermal conductivity, used in optical applications and as a heatsink substrate. Explaining many of its properties depends on an accurate description of its lattice dynamics, which have thus far only been captured in the quasiharmonic approximation. In this work, we show that anharmonic effects have a considerable impact on its phase stability and transport properties, since they are much stronger in the rocksalt phase. We construct a theoretical pressure-temperature phase diagram of AlN, demonstrating that the rocksalt phase is stabilized by increasing temperature, with respect to the wurtzite phase. We recover the thermal conductivity of the wurtzite phase ( $320 \mathrm{Wm}^{-1} \mathrm{~K}^{-1}$ under ambient conditions) and compute the hitherto unknown thermal conductivity of the rocksalt phase $\left(81 \mathrm{Wm}^{-1} \mathrm{~K}^{-1}\right)$. We also show that the electronic band gap decreases with temperature. These findings provide further evidence that anharmonic effects cannot be ignored in simulations of materials intended for high-temperature applications.
\end{abstract}

DOI: 10.1103/PhysRevB.94.104305

\section{INTRODUCTION}

Aluminium nitride (AIN) in the wurtzite structure is a wide-band-gap $(6.3 \mathrm{eV})$ semiconductor [1] and is, at the same time, one of the best nonmetallic thermal conductors; at room temperature, its thermal conductivity is $319 \mathrm{Wm}^{-1} \mathrm{~K}^{-1}$, about $80 \%$ of the thermal conductivity of copper [2]. Its thermal expansion is low, and similarly to that of silicon, and it has a high hardness. These properties are important factors in its use as a heat-sink substrate for electronic semiconductor devices [2].

Like the other group III nitrides, AlN undergoes a pressureinduced phase transition from the wurtzite structure at ambient pressure to the rocksalt structure at high pressures and temperatures. At room temperature, the transition has been experimentally shown to occur between $12 \mathrm{GPa}$ [3] and $16 \mathrm{GPa}$ [4]. Theoretical calculations at zero temperature recover this transition and have identified competing transition pathways from wurtzite to rocksalt: a uniaxial strain resulting in a tetragonal transition state, and a shear stress resulting in a hexagonal transition state [5-7], with the hexagonal pathway favored by AlN. There is also a zinc-blende phase that can be produced experimentally, but we do not include it in our calculations since it is metastable [8], and we are interested in equilibrium phases.

However, the high-temperature behavior of AlN has received less attention. Calculations within the quasiharmonic approximation (QHA)[9-13] placed the transition pressure between 12.9 $\mathrm{GPa}$ [11] and $17 \mathrm{GPa}$ [9], depending on the details of the calculation. They also suggest that the rocksalt structure is stabilized by temperature, but the extent of this effect cannot be extrapolated from the QHA, which breaks down at high temperatures due to anharmonic effects; Schmerler and Kortus [12] demonstrate that the temperature limit is approximately $2200 \mathrm{~K}$.

The behavior of the $c / a$ ratio with respect to volume is unusual for wurtzite-type crystals. If the pressure is increased along an isotherm, $c / a$ increases, but if the temperature is increased along an isobar, $c / a$ shows the opposite behavior and decreases - this can cause problems if only zero-temperature structures are used for QHA calculations [12]. In practice, this problem can be overcome by interpolating $c / a$ as a function of temperature and volume. The larger problem is that phases which are in reality dynamically unstable at zero temperature but dynamically stable at some finite temperature will never be stabilized by temperature within the harmonic approximation. By employing the temperature-dependent effective potential method [14-16], we overcome both of these issues and can compute phonon spectra which include high-temperature anharmonicity.

Lattice vibrations are important not only for determining phase stability [17]; in semiconductors, they are responsible for most of the heat transport, which is critical in the case of $T i_{1-x} A l_{x} N$ alloys. The thermal conductivity of the wurtzite phase of AlN has been measured but is unknown for rocksalt, which is metastable at ambient conditions and can only be grown as a thin film, for which it is difficult to measure the thermal conductivity. It is important to know the thermal conductivity of the rocksalt phase for high-temperature applications and because $\mathrm{AlN}$ is the decomposition product of many useful materials such as $T i_{1-x} A l_{x} N$ alloys [11].

AlN is also an important semiconductor in its own right. When doped with $\mathrm{Si}$, it can be used in ultraviolet lightemitting diodes with a wavelength of $210 \mathrm{~nm}$ [18]. However, experiments have shown that the band gap can be reduced by increasing the temperature for pure AIN [19], potentially offering a method of controlling the band gap without doping.

Large single crystals of AIN are difficult to fabricate due to the required process control at high temperatures [20,21], but $a b$ initio simulation provides us with a probe of its properties at extreme conditions. The aims of this paper are threefold. First, we want to ascertain the effect of anharmonicity on the phase stability of AlN, beyond the QHA. Second, we want to determine the effect of anharmonicity on the unusually high 
thermal conductivity of AlN. Finally, we investigate the effect of temperature on the electronic band gap, including the effects of thermal lattice disorder.

\section{METHODS}

To determine the pressure-temperature phase diagram of AlN we need to accurately describe the vibrational degrees of freedom and determine the corresponding vibrational entropy. We employ a method that provides a superior description of the finite-temperature behavior to the QHA. Our method uses Born-Oppenheimer molecular dynamics (BOMD) to more effectively sample the potential energy surface. A general problem with the QHA is the appearance of negative branches in the phonon dispersion at large volumes [12], as a result of the shift in the equilibrium volume as a function of temperature; BOMD does not suffer from this problem.

Our approach to this problem is to map the lattice dynamics of the AlN system to a model Hamiltonian, described by the temperature-dependent effective potential method (TDEP) [14-16] of the following form:

$$
\begin{aligned}
H= & U_{0}+\sum_{i} \frac{\mathbf{p}_{i}^{2}}{2 m_{i}}+\frac{1}{2 !} \sum_{i j \alpha \beta} \Phi_{i j}^{\alpha \beta} u_{i}^{\alpha} u_{j}^{\beta} \\
& +\frac{1}{3 !} \sum_{i j k \alpha \beta \gamma} \Psi_{i j k}^{\alpha \beta \gamma} u_{i}^{\alpha} u_{j}^{\beta} u_{k}^{\gamma} .
\end{aligned}
$$

Here, $m_{i}, p_{i}$, and $u_{i}$ are the mass, momentum, and displacement from equilibrium of atom $i ; \alpha \beta \gamma$ corresponds to Cartesian components; and $\Phi$ and $\Psi$ are the secondand third-order effective interatomic force constants (IFCs). $U_{0}$ is the temperature-dependent ground-state energy of the model system. The IFCs are denoted as effective since they implicitly contain all orders of anharmonicity and are explicitly temperature dependent.

We performed canonical (NVT) BOMD using the projector augmented wave (PAW) method [23] as implemented in the Vienna $a b$ initio Simulation Package (VASP) [24-27]. The electronic exchange-correlation energy was calculated within the generalized gradient approximation, using the PerdewBurke-Ernzerhof (PBE) functional [28]. For the rocksalt AIN phase we employed a $5 \times 5 \times 5$ (250 atom) supercell and a $4 \times 4 \times 4$ (256 atoms) supercell for the wurtzite structure. We ran Born-Oppenheimer molecular dynamics on a grid of 6 temperatures from $300 \mathrm{~K}$ to $3100 \mathrm{~K}$, with five volumes for each temperature for each phase. We also optimized the $c / a$ ratio, using a grid of five values of $c / a$ for each temperature and volume. The temperature was controlled using a Nosé thermostat [29]. The plane-wave energy cutoff was set to $600 \mathrm{eV}$, and the Brillouin zone sampled at the $\Gamma$ point during molecular dynamics simulations for a total of 10000 time steps of $0.5 \mathrm{fs}$ each. The initial state of the supercell was prepared in a thermally excited state using TDEP force constants to minimize or completely remove the equilibration time [31]. Energies were later recomputed for 300 uncorrelated snapshots using a $3 \times 3 \times 3$ Monkhorst-Pack [32] grid of $k$ points. We also used the small-displacement method to calculate the phonon spectra in the QHA for comparison.
In the TDEP formalism the Helmholtz free energy is given by [15]

$$
F_{\mathrm{TDEP}}=U_{0}+F_{\mathrm{vib}},
$$

where $F_{\text {vib }}$ is calculated from the explicitly temperature and volume-dependent phonon density of states. From this, we calculate the pressure as $P=-d F / d V$ and obtain Gibbs free energy as $G=U_{0}+F_{\text {vib }}+P V$. For the wurtzite phase, the optimal $c / a$ ratio is determined from the Helmholtz free energy at each volume and temperature.

In order to compute the thermal conductivity, we solved the Boltzmann transport equation (BTE) beyond the relaxation time approximation [33-36]. The phonon frequencies, group velocities, and scattering rates were calculated at each temperature, since at every temperature the IFCs, volume, and $c / a$ ratio differ. The BTE was solved on a $25 \times 25 \times 25 q$-point grid on which momentum is exactly conserved, and the tetrahedron approach [23] was employed for energy conservation.

\section{RESULTS AND DISCUSSION}

\section{A. Phase stability}

In Fig. 1, we present the calculated pressure-temperature phase diagram for AIN, including the regions of stability (i.e., lowest Gibbs free energy) for wurtzite-AlN (green) and rocksalt-AlN (yellow). The dashed curve denotes the intersection of the Gibbs free-energy surfaces for the two phases within the QHA and are a marked contrast with the analogous TDEP curve at high temperatures. At temperatures below $1500 \mathrm{~K}$ anharmonic effects have negligible impact on

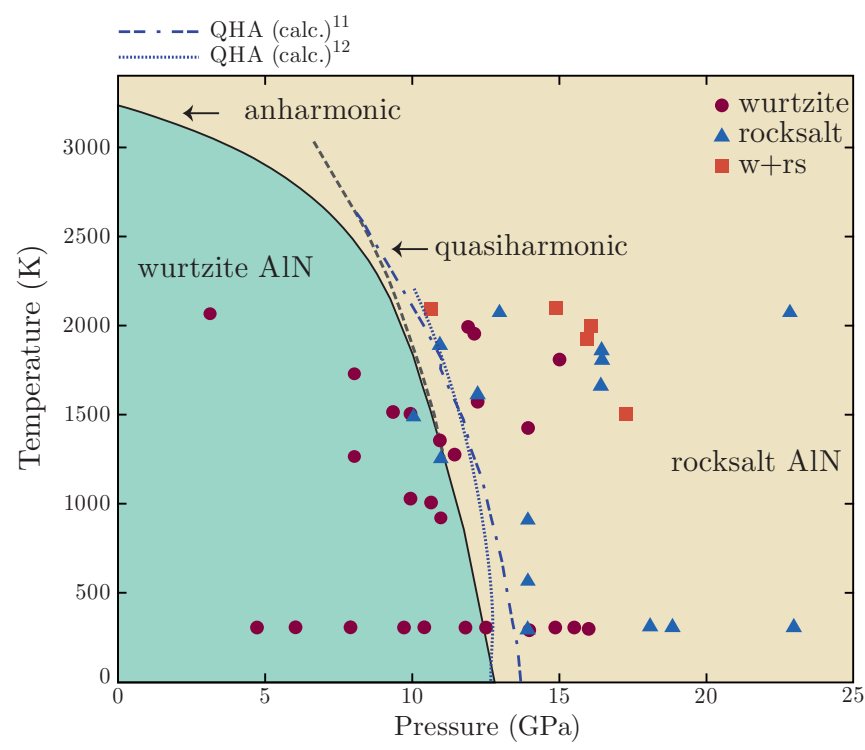

FIG. 1. Temperature-pressure phase diagram of AlN. Experimental data are collected from Refs. [1,3,4,11,22]. Theoretical data are extracted from Refs. [11,12] and show the difference in the boundary line between the wurtzite and rocksalt phases depending on a choice of the functional. Our anharmonic phase boundary is in good agreement with previous QHA calculations at low temperatures. There is considerable uncertainty in the actual transition boundary, as evidenced by the experimental data points, and it is sensitive to the experimental details and in the case of simulations, the choice of exchange-correlation functional. 


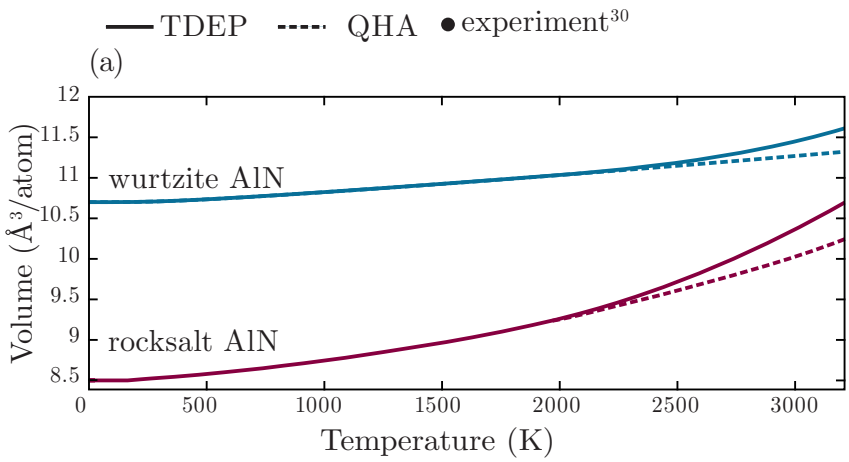

(b)
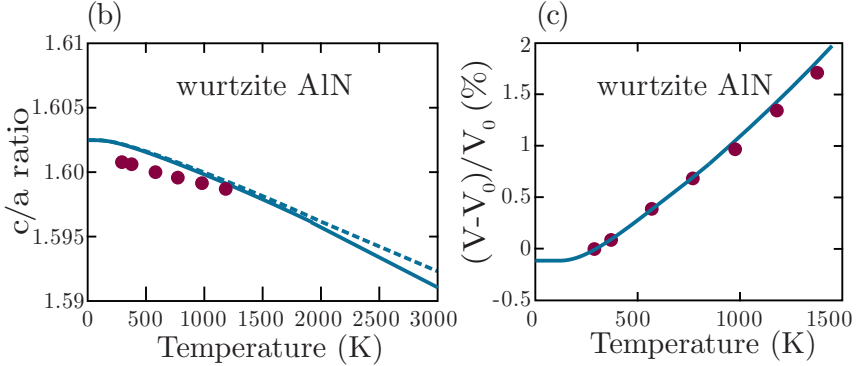

FIG. 2. (a) Comparison of volume as a function of temperature computed anharmonically using TDEP and quasiharmonically. (b) $c / a$ ratio as a function of temperature for wurtzite AlN, computed anharmonically and quasiharmonically. Red points are from experiments [30]. (c) Comparison of the relative volume expansion as a function of temperature with experimental data [30]. $V_{0}$ is the room temperature equilibrium volume.

phase stability, but at higher temperatures they rapidly become important. The anharmonicity pushes the phase boundary to lower pressure at high temperature to the extent that the rocksalt phase is stabilized by temperature at the lower end of the pressure scale. The melting temperature of AlN is not known, but predictions place it at around $3000 \mathrm{~K}$ [9]. Nonetheless, the possibility remains that AlN might melt from the rocksalt phase.

Figure 2 demonstrates the contrast between the thermal expansion computed in the QHA and using the fully anharmonic approach of TDEP. Below $2000 \mathrm{~K}$, both methods agree well but diverge at higher temperatures. This is because the zero-temperature equilibrium volume is generally used in quasiharmonic calculations, and at high temperatures thermal expansion causes enough of a volume change to invalidate this approach. Our results agree with previous calculations using the QHA and with experimental data at temperatures below $2000 \mathrm{~K}[1,3,4,9,11,12,22]$. In the lower panel of Fig. 2, the $c / a$ ratio is plotted as a function of temperature. The experimental values are nonequilibrium and do not agree exactly, but they qualitatively demonstrate the way in which the $c / a$ ratio varies with temperature. At high temperatures the difference between the QHA and TDEP curves becomes pronounced, suggesting that the QHA does not capture this phenomenon. Therefore, although it has already been shown that $c / a$ varies with pressure and temperature [12,30,37], we demonstrate that anharmonic effects cannot be dismissed and have an impact on phase stability at high temperatures.

Comparing the behavior of the volumes of the rocksalt and wurtzite phases as a function of temperature reveals a striking
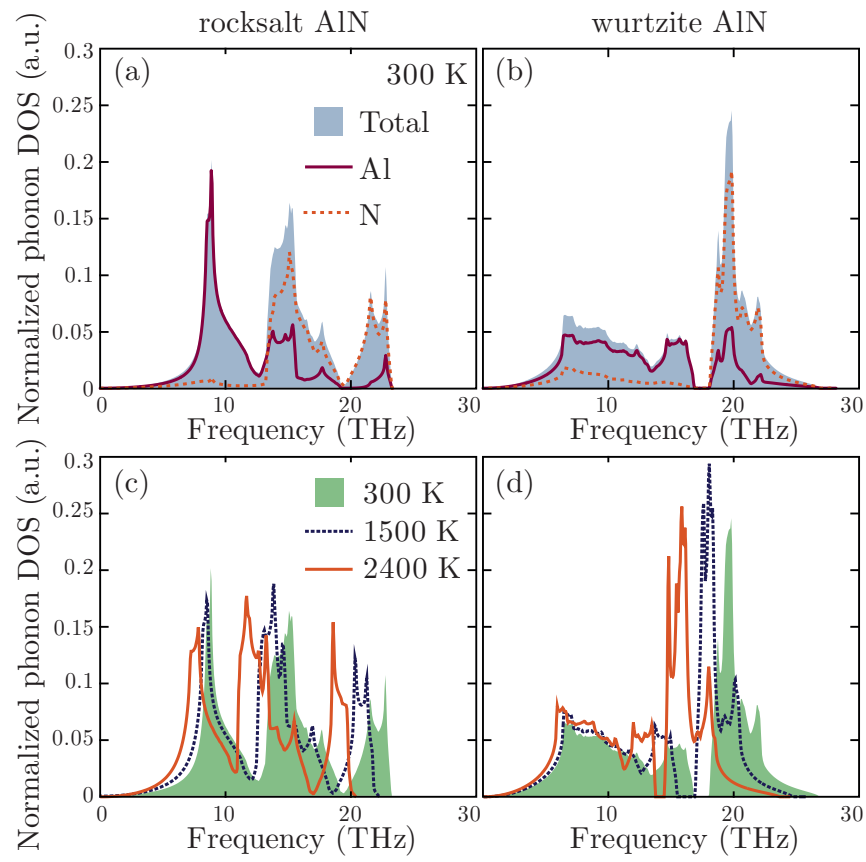

FIG. 3. Total phonon density of states and partial phonon density of states per $\mathrm{Al}$ and $\mathrm{N}$ atoms at room temperature for (a) rocksalt AlN and (b) wurtzite AlN. Total phonon density of states as a function of temperature (c) rocksalt AlN (d) wurtzite AlN.

difference. In Fig. 2(a) we see that anharmonicity has a much stronger influence on the rocksalt phase than on the wurtzite phase, so much so that rocksalt AlN is dynamically unstable in the QHA [12] for reasons discussed earlier. Anharmonic effects stabilize it at high temperatures and lower pressures.

The effect of anharmonicity can be highlighted by examining the individual contributions of $\mathrm{Al}$ and $\mathrm{N}$ atoms to the phonon density of states and by observing the effect of temperature on the total phonon density of states. In Figs. 3(a) and 3(b) we show the partial phonon density of states, decomposed into contributions from $\mathrm{Al}$ and $\mathrm{N}$ atoms. In general, the $\mathrm{Al}$ atoms contribute more at low frequencies, which is unsurprising given their larger mass. However, the contribution of $\mathrm{N}$ atoms to the low frequencies in wurtzite [Fig. 3(b)] is a much larger proportion of the total, while the the high-frequency optical modes in wurtzite are dominated to a much greater extent by $\mathrm{N}$ vibrational modes.

In Figs. 3(c) and 3(d), the effect of temperature-induced anharmonicity can be seen. The rocksalt phase [Fig. 3(c)] is subject to a higher degree of anharmonicity because the shift in the phonon density of states with increasing temperature is more pronounced, and the acoustic modes of the rocksalt phase soften more rapidly with temperature than for the wurtzite phase. When considering phase stability, the dominant contribution to the free energy arises from the acoustic modes, and thus the enhanced acoustic softening in the case of rocksalt explains its metastability relative to wurtzite at high temperatures. Examining the partial contributions, the spectrum for wurtzite contains states with a higher degree of hybridization, whereas the states in the rocksalt phase are more separated, allowing more rapid softening for the latter. 
The strong anharmonicity in the cubic phase in comparison to wurtzite can be explicitly illustrated using the third-order effective IFCs. In perfectly harmonic solids the phonons are noninteracting quasiparticles with well-defined frequencies. This is not true in general, since anharmonicity includes phonon-phonon interactions. This can be quantified with the frequency-dependent phonon self-energy, $\Sigma=\Delta+i \Gamma$, which will shift and broaden the phonon spectrum [38]:

$$
\begin{aligned}
\Gamma_{\mathbf{q} s}(\Omega)= & \sum_{s^{\prime} s^{\prime \prime}} \frac{\hbar \pi}{16} \frac{V}{(2 \pi)^{3}} \iint_{\mathrm{BZ}}\left|\Psi_{s s^{\prime} s^{\prime \prime}}^{\mathbf{q} \mathbf{q}^{\prime} \mathbf{q}^{\prime \prime}}\right|^{2} \Delta_{\mathbf{q} \mathbf{q}^{\prime} \mathbf{q}^{\prime \prime}} \\
& \times\left[\left(n_{\mathbf{q}^{\prime} s^{\prime}}+n_{\mathbf{q}^{\prime \prime} s^{\prime \prime}}+1\right) \delta\left(\Omega-\omega_{\mathbf{q}^{\prime} s^{\prime}}-\omega_{\mathbf{q}^{\prime \prime} s^{\prime \prime}}\right)\right. \\
& \left.+2\left(n_{\mathbf{q}^{\prime} s^{\prime}}-n_{\mathbf{q}^{\prime \prime} s^{\prime \prime}}\right) \delta\left(\Omega-\omega_{\mathbf{q}^{\prime} s^{\prime}}+\omega_{\mathbf{q}^{\prime \prime} s^{\prime \prime}}\right)\right] d \mathbf{q}^{\prime} d \mathbf{q}^{\prime \prime}
\end{aligned}
$$

and the real part of the self-energy is given by a KramersKronig transformation:

$$
\Delta(\Omega)=\frac{1}{\pi} \int \frac{\Gamma(\omega)}{\omega-\Omega} d \omega .
$$

Here $\mathbf{q}$ denotes a phonon wave vector and $s$ the phonon mode and $n_{\mathbf{q} s}$ is the equilibrium occupation number, $\omega_{\mathbf{q} s}$, is the frequency of mode $s$ at wave vector q. Equation (3) ensures momentum and energy conservations through $\Delta_{\mathbf{q q} \mathbf{q}^{\prime} \mathbf{q}^{\prime \prime}}$ and $\delta$ functions, respectively. $\Psi_{s s^{\prime} s^{\prime \prime}}^{\mathbf{q q} \mathbf{q}^{\prime \prime}}$ is the Fourier-transformed third-order force constant and $\hbar \Omega$ is the probing energy. From the self-energy, one can calculate the one-neutron cross section or the phonon line shape using

$$
\sigma_{\mathbf{q} s}(\Omega) \propto \frac{2 \omega_{\mathbf{q} s} \Gamma_{\mathbf{q} s}(\Omega)}{\left[\Omega^{2}-\omega_{\mathbf{q} s}^{2}-2 \omega_{\mathbf{q} s} \Delta_{\mathbf{q} s}(\Omega)\right]^{2}+4 \omega_{\mathbf{q} s}^{2} \Gamma_{\mathbf{q} s}^{2}(\Omega)} .
$$

This phonon line shape is shown in Fig. 4. The amount of broadening at zero temperature, from the zero point motion, is minimal for both phases. At $2400 \mathrm{~K}$, the broadening and therefore the anharmonicity is much stronger in the rocksalt phase. In the wurtzite phase at $2400 \mathrm{~K}$ [Fig. 4(d)], the optic modes are subject to the highest degree of broadening, but in the rocksalt phase [Fig. 4(c)], the acoustic modes are also broadened a great deal at the low-frequency end of the
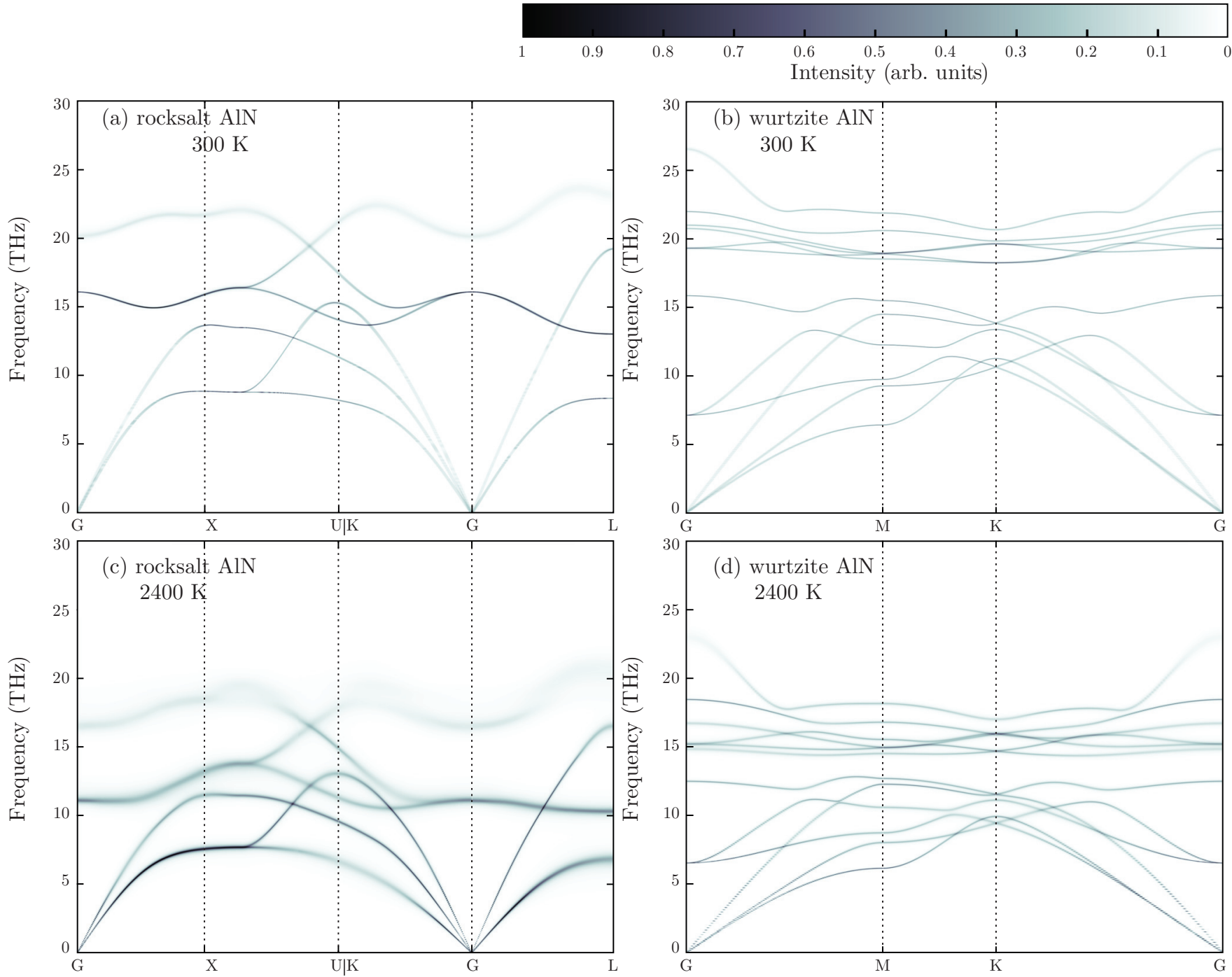

FIG. 4. Phonon lineshapes for rocksalt (left) and wurtzite (right) AlN at $300 \mathrm{~K}$ and $2400 \mathrm{~K}$ and ambient pressure. There is insignificant broadening at $300 \mathrm{~K}$. From the broadening at $2400 \mathrm{~K}$, it is clear that the anharmonicity in the rocksalt phase is significantly stronger than for wurtzite across all branches. 
spectrum, particularly in the 2 - to $7-\mathrm{THz}$ range. Note that the phonon dispersions at $2400 \mathrm{~K}$ include both the effects of thermal expansion and intrinsic anharmonicity. Since AIN is a polar semiconductor, the Born effective charges effect the vibrational optical modes around $\Gamma$ point splitting the frequencies of those modes parallel and perpendicular to the electric field, i.e., LO-TO splitting, splitting between longitudinal optical and transverse optical frequencies. These quantities are calculated from density functional perturbation theory [39], implemented in VASP, with the volumes chosen to correspond to the respective temperatures. For example, at $300 \mathrm{~K}$ the diagonal elements of the dielectric tensor for the rocksalt phase are $\epsilon_{x x}=\epsilon_{y y}=\epsilon_{z z}=5.2356$, compared with $\epsilon_{x x}$ $=\epsilon_{y y}=4.1013$ and $\epsilon_{z z}=4.3547$ for wurtzite $($ since $c / a \neq 1)$. The Born effective charges are $+2.997 e$ and $-2.997 e$ for $\mathrm{Al}$ and $\mathrm{N}$ atoms in the rocksalt structure, respectively, and $+2.487 e$ for the $x$ and $y$ components, $+2.6656 e$ for the $z$ component, $-2.487 e$ for the $x$ and $y$ components, and $+2.6656 e$ for $z$ component for wurtzite. These values are in a good agreement with previously reported values $[40,41]$.

\section{B. Thermal transport}

Since AlN is a wide-band-gap material, thermal transport is dominated by phonons. Intuitively, the difference in anharmonic behavior between the phases should lead to a large difference in their thermal conductivity. From the BTE, the diagonal components of the thermal conductivity tensor are given by

$$
\kappa_{\alpha \alpha}=\frac{1}{V} \sum_{\mathbf{q} s} C_{\mathbf{q} s} v_{\alpha \mathbf{q} s}^{2} \tau_{\alpha \mathbf{q} s},
$$

where $v_{\alpha \mathbf{q} s}$ is the phonon group velocity of mode $\mathbf{q} s, \tau_{\alpha \mathbf{q} s}$ is the phonon lifetime for transport in direction $\alpha$, and $C_{\mathbf{q} s}$ is the mode specific heat. The thermal conductivity tensor in rocksalt systems is diagonal with $\kappa_{x x}=\kappa_{y y}=\kappa_{z z}$, and for hexagonal structures there is an out-of-plane component $\kappa_{z z}$ and an inplane component $\kappa_{x x}=\kappa_{y y}$. The lattice thermal conductivities for both phases are plotted as a function of temperature in Fig. 5. We first note that the in-plane and out-of-plane components are very similar, because the wurtzite AIN $c / a$ ratio is weakly temperature dependent. Our values are in good agreement with existing experimental and theoretical results at room temperature [42], except for the point at $100 \mathrm{~K}$. This low-temperature divergence is expected because a real crystal contains defects and grain boundaries, which have a more significant impact (i.e., scatter phonons more) at low temperatures [35]. Rocksalt AlN is metastable and can be synthesized only as a thin film, so measurements of thermal conductivity have not yet been reported. We note that the larger anharmonicity of the rocksalt phase has a significant impact on the thermal conductivity: At $300 \mathrm{~K}$, it is $81 \mathrm{Wm}^{-1} \mathrm{~K}^{-1}$ in the rocksalt phase and $320 \mathrm{Wm}^{-1} \mathrm{~K}^{-1}$ in the wurtzite $\left(319 \mathrm{Wm}^{-1} \mathrm{~K}^{-1}\right.$ from experiments [2]), a difference of a factor of 4 . This is contrary to the intuition that a simpler, more symmetric crystal structure should have a higher thermal conductivity [43,44].

To explain the origin of the difference in thermal conductivity, we performed a model calculation with the third-order IFCs set to 1 for both phases. The difference in thermal conductivity between the phases then reduces to less than $1 \%$. This allows us

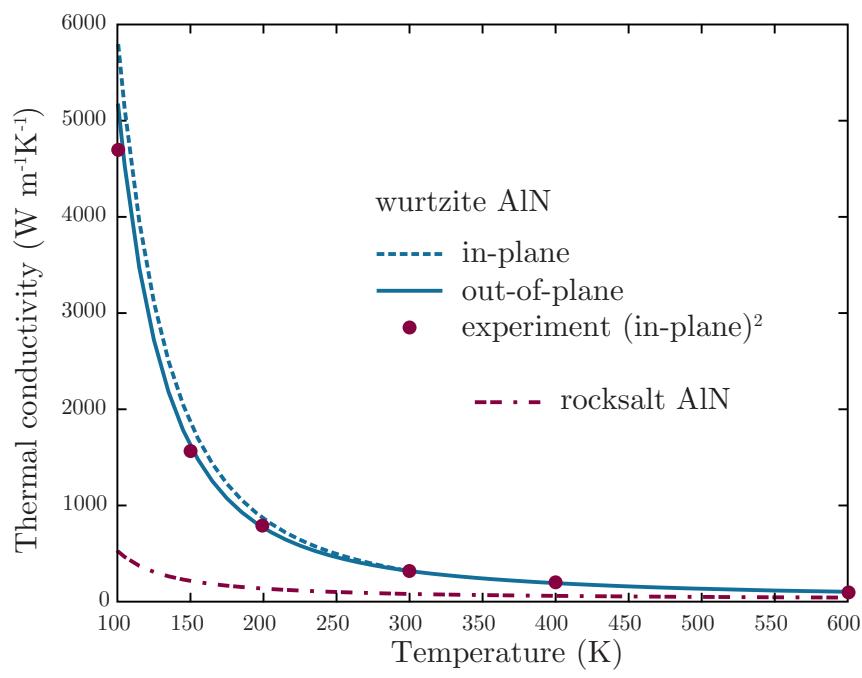

FIG. 5. Thermal conductivity of rocksalt and wurtzite phases as a function of temperature at ambient pressure. Experimental data are taken from Ref. [2] correspond to in-plane thermal conductivity for the wurtzite phase.

to conclude that the harmonic parts of the thermal conductivity (scattering phase space, heat capacities, and group velocities) in the rocksalt and wurtzite phases are more or less the same, and the difference arises from the strength of the anharmonic three-body interactions, which are about twice as strong in the rocksalt phase as compared with the wurtzite.

The cumulative part of the lattice thermal conductivity can be measured as a function of the phonon mean free path in experiments [45-47]. The mean free path of a phonon in mode $\mathbf{q} s$ is defined as $\left|\mathbf{v}_{\mathbf{q} s}\right| \tau_{\alpha \mathbf{q} s}$. The cumulative thermal conductivity can then be computed as a sum of the fraction of heat that is carried by phonons with mean free paths smaller than $l$ [36]:

$$
\kappa_{\alpha \alpha}^{\mathrm{acc}}(l)=\frac{1}{V} \sum_{\mathbf{q} s} C_{\mathbf{q} s} v_{\alpha \mathbf{q} s}^{2} \tau_{\alpha \mathbf{q} s} \Theta\left(l-\left|\mathbf{v}_{\mathbf{q} s}\right| \tau_{\alpha \mathbf{q} s}\right),
$$

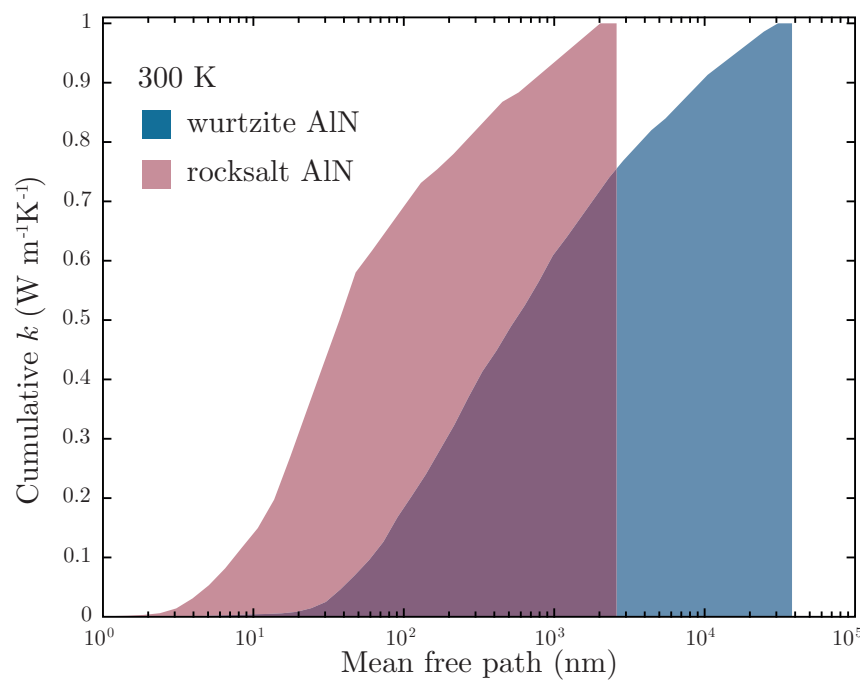

FIG. 6. Cumulative thermal conductivity as a function of the mean free path for the rocksalt and wurtzite phases of AlN at room temperature. 
where $\Theta$ is the Heaviside step function. The normalized cumulative room temperature thermal conductivity (calculated from TDEP force constants) is shown in Fig. 6. The mean free path of the rocksalt phase is 15 times shorter than for the wurtzite phase, implying that phonon lifetimes in the rocksalt phase are shorter, which indicates that it is more anharmonic.

\section{Electronic properties}

At finite temperatures the lattice will be perturbed by vibrations. This deviation from a perfect lattice will lead to shifts and broadening of the electronic bands. To quantify this, we use the electronic structure from uncorrelated snapshots extracted from molecular dynamics simulations and average them, relying on the ergodic principle, i.e., that the time average will be equivalent to an ensemble average. We used the band unfolding technique $[48,49]$ to reduce the band structures and calculate the spectral functions. To compute the band structure, we combined 100 thermally excited samples generated as discussed in the methods section.

Examining the spectral functions for rocksalt and wurtzite $\mathrm{AlN}$ at $300 \mathrm{~K}$ and $2400 \mathrm{~K}$ (Fig. 7), it can be seen that thermal disorder severely broadens and shifts the Kohn-Sham eigenstates. In principle, we need to consider many-body effects to obtain accurate band gaps, since in the GGA formalism, they are consistently underestimated; however, the DFT results are still qualitatively useful for determining trends [50]. Computing the direct band gap at different temperatures (Fig. 8), temperature-induced band gap narrowing can be observed. These calculations are consistent with cathodoluminescence measurements, which show a band-gap decrease of $0.1 \mathrm{eV}$ between $0 \mathrm{~K}$ and $300 \mathrm{~K}$ [19], which can be attributed to thermal expansion. The rocksalt phase has a band gap that is about $1 \mathrm{eV}$ larger at $0 \mathrm{~K}$, although the gaps become similar as the temperature increases. It is also interesting to note that at low temperature, the indirect band gap for rocksalt $\mathrm{AlN}$ is lower, but at high temperatures, the direct band gap becomes smaller.

The finite temperature spectral functions in Fig. 8 are compared with zero-temperature dispersions at the same volume (red lines), i.e., including only the effect of thermal expansion. We can thus qualitatively see the effect of thermal lattice disorder on the electronic band structure: They are distorted as well as broadened.
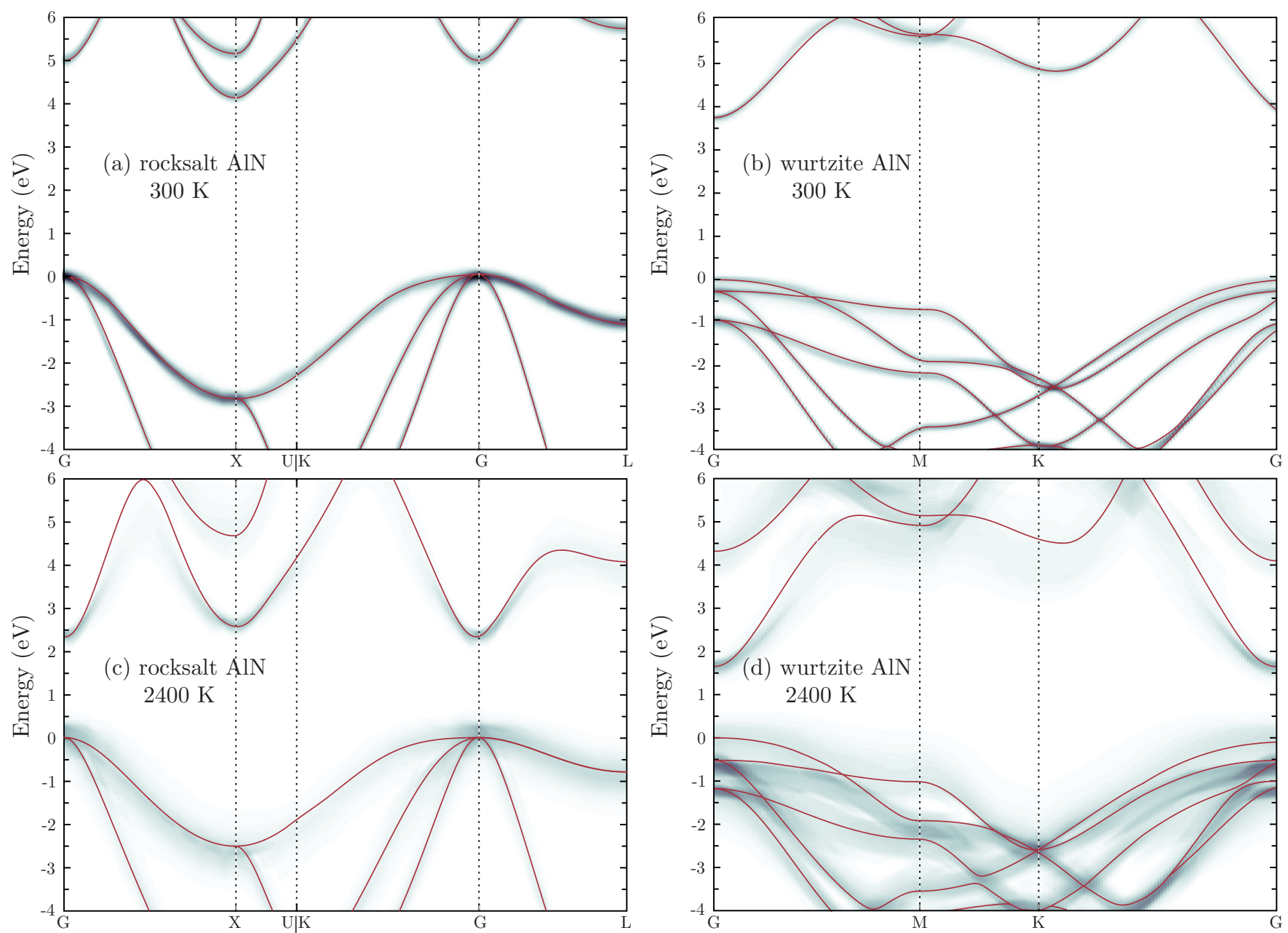

FIG. 7. Spectral function for the rocksalt phase at $300 \mathrm{~K}$ and $2400 \mathrm{~K}$ and ambient pressure, showing the broadening of electronic bands due to thermal disorder. The lines indicate the band structures computed at zero temperature for thermally expanded cells in the absence of the thermal atomic displacements and compares them with band structures that include both volume expansion and thermal disorder. 


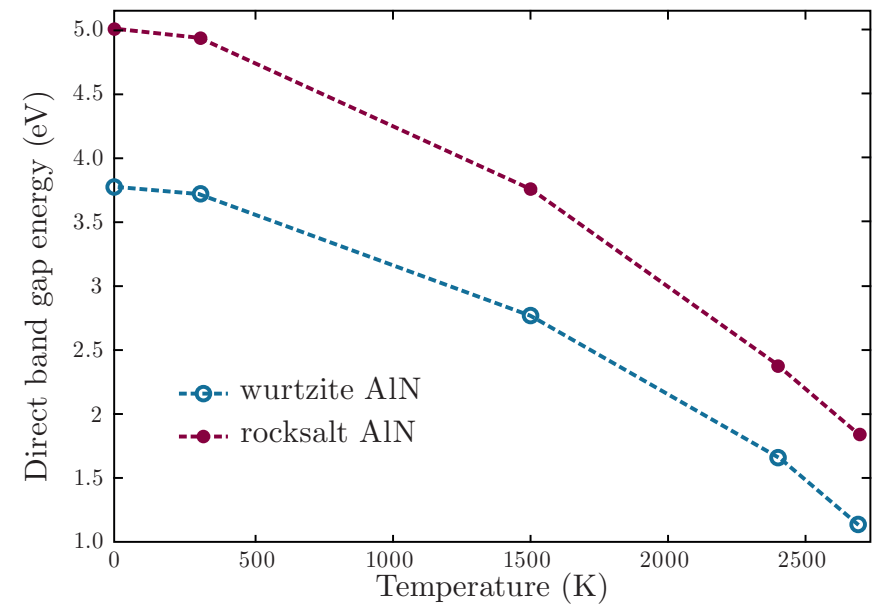

FIG. 8. Direct electronic band gap as a function of temperature for the rocksalt and wurtzite phases at ambient pressure.

\section{CONCLUSIONS}

We have argued that anharmonicity is extremely important in the high-temperature description of AlN and has a number of direct and indirect consequences in its physical properties. Rocksalt AlN is much more anharmonic than wurtzite, and, as a result, the former is stabilized relative to the latter as the temperature increases to the extent that there may be a temperature-induced phase change from wurtzite to rocksalt at ambient pressure, assuming melting does not occur first. The anharmonicity beyond the QHA also has a significant effect on the thermal expansion, with the rocksalt phase undergoing more thermal expansion at high temperatures. It also influences the temperature-induced change in the $c / a$ ratio for the wurtzite phase and cannot be ignored under hightemperature conditions.

We have achieved a good agreement with experiments for the thermal conductivity of the wurtzite phase between $100 \mathrm{~K}$ and $600 \mathrm{~K}$. We have also used the predictive power of the TDEP method to compute the thermal conductivity of the rocksalt phase, which has a much lower thermal conductivity, suggesting that if there is a premelting phase transition at high temperatures, the properties of AlN will change precipitously. By looking at the thermally disordered band structure, we observe a decrease in the band gap of both phases as the temperature increase and a change from an indirect semiconductor to a direct semiconductor in the case of rocksalt AlN.

\section{ACKNOWLEDGMENTS}

Support from the Swedish Research Council (VR programs 2015-04391 and 621-2012-4401), Swedish Foundation for Strategic Research (SSF program SRL10-0026), and VINNOVA [M-Era.net Project No. 2013-02355 (MC2)] is acknowledged. O.H. acknowledges the Swedish Research Council VR program 637-2013-7296. Z.R. acknowledges the Swedish Foundation for Strategic Research (SSF) program FUNCASE. I.A.A. is grateful for the support from the Ministry of Education and Science of the Russian Federation in the framework of Increase Competitiveness Program of NUST "MISIS" (No. K2-2016-013). All calculations were performed using the supercomputer resources of the SwedishNational Infrastructure for Computing (SNIC), National Supercomputer Centre at Linköping University (NSC) and the PDC Center for High Performance Computing at the KTH Royal Institute of Technology.
[1] Q. Xia, H. Xia, and A. L. Ruoff, J. Appl. Phys. 73, 8198 (1993).

[2] G. A. Slack, R. Tanzilli, R. Pohl, and J. Vandersande, J. Phys. Chem. Solids 48, 641 (1987).

[3] M. Schwarz, M. Antlauf, S. Schmerler, K. Keller, T. Schlothauer, J. Kortus, G. Heide, and E. Kroke, High Press. Res. 34, 22 (2013).

[4] L. Bayarjargal and B. Winkler, Appl. Phys. Lett. 100, 021909 (2012).

[5] A. M. Saitta and F. Decremps, Phys. Rev. B 70, 035214 (2004).

[6] J. Cai and N. Chen, Phys. Rev. B 75, 134109 (2007).

[7] R. F. Zhang and S. Veprek, Acta Mater. 57, 2259 (2009).

[8] I. Petrov, E. Mojab, R. C. Powell, J. E. Greene, L. Hultman, and J. Sundgren, Appl. Phys. Lett. 60, 2491 (1992).

[9] A. Siegel, K. Parlinski, and U. D. Wdowik, Phys. Rev. B 74, 104116 (2006).

[10] F. J. Manjón, D. Errandonea, A. H. Romero, N. Garro, J. Serrano, and M. Kuball, Phys. Rev. B 77, 205204 (2008).

[11] N. Norrby, H. Lind, G. Parakhonskiy, M. P. Johansson, F. Tasnádi, L. S. Dubrovinsky, N. Dubrovinskaia, I. A. Abrikosov, and M. Odén, J. Appl. Phys. 113, 053515 (2013).

[12] S. Schmerler and J. Kortus, Phys. Rev. B 89, 064109 (2014).

[13] J. C. Nipko and C.-K. Loong, Phys. Rev. B 57, 10550 (1998).
[14] O. Hellman, I. A. Abrikosov, and S. I. Simak, Phys. Rev. B 84, 180301 (2011).

[15] O. Hellman, P. Steneteg, I. A. Abrikosov, and S. I. Simak, Phys. Rev. B 87, 104111 (2013).

[16] O. Hellman and I. A. Abrikosov, Phys. Rev. B 88, 144301 (2013).

[17] N. Shulumba, O. Hellman, L. Rogström, Z. Raza, F. Tasnádi, I. A. Abrikosov, and M. Odén, Appl. Phys. Lett. 107, 231901 (2015).

[18] Y. Taniyasu, M. Kasu, and T. Makimoto, Nature 441, 325 (2006).

[19] E. Silveira, J. Freitas, S. Schujman, and L. Schowalter, J. Cryst. Growth 310, 4007 (2008).

[20] G. A. Slack and T. F. McNelly, J. Cryst. Growth 34, 263 (1976).

[21] G. A. Slack and T. F. McNelly, J. Cryst. Growth 42, 560 (1977).

[22] H. Vollstadt, E. Ito, M. Akaishi, S. Akimoto, and O. Fukunaga, Proc. Jpn. Acad. B 66, 7 (1990).

[23] P. E. Blöchl, Phys. Rev. B 50, 17953 (1994).

[24] G. Kresse and J. Hafner, Phys. Rev. B 48, 13115 (1993).

[25] G. Kresse and J. Furthmüller, Phys. Rev. B 54, 11169 (1996).

[26] G. Kresse and J. Furthmüller, Comput. Mater. Sci. 6, 15 (1996).

[27] M. Gajdoš, K. Hummer, G. Kresse, J. Furthmüller, and F. Bechstedt, Phys. Rev. B 73, 045112 (2006). 
[28] J. P. Perdew, K. Burke, and M. Ernzerhof, Phys. Rev. Lett. 77, 3865 (1996).

[29] S. Nosé, Prog. Theor. Phys. Suppl. 103, 1 (1991).

[30] H. Iwanaga, A. Kunishige, and S. Takeuchi, Mater. Sci. 35, 2451 (2000).

[31] P. Steneteg, O. Hellman, O. Y. Vekilova, N. Shulumba, F. Tasnádi, and I. A. Abrikosov, Phys. Rev. B 87, 094114 (2013).

[32] H. J. Monkhorst and J. D. Pack, Phys. Rev. B 13, 5188 (1976).

[33] M. Omini and A. Sparavigna, Physica B: Condens Matter 212, 101 (1995).

[34] D. A. Broido, L. Lindsay, and A. Ward, Phys. Rev. B 86, 115203 (2012).

[35] L. Lindsay, D. A. Broido, and T. L. Reinecke, Phys. Rev. B 87, 165201 (2013).

[36] O. Hellman and D. A. Broido, Phys. Rev. B 90, 134309 (2014).

[37] N. E. Christensen and I. Gorczyca, Phys. Rev. B 47, 4307 (1993).

[38] R. A. Cowley, Rep. Prog. Phys. 31, 123 (1968).

[39] S. Baroni and R. Resta, Phys. Rev. B 33, 7017 (1986).

[40] K. Karch and F. Bechstedt, Phys. Rev. B 56, 7404 (1997).
[41] A. R. Goñi, H. Siegle, K. Syassen, C. Thomsen, and J.-M. Wagner, Phys. Rev. B 64, 035205 (2001).

[42] A. Jacquot, B. Lenoir, A. Dauscher, P. Verardi, F. Craciun, M. Stölzer, M. Gartner, and M. Dinescu, Appl. Surf. Sci. 186, 507 (2002).

[43] G. A. Slack, J. Phys. Chem. Solids 34, 321 (1973).

[44] G. A. Slack, Solid State Physics (Academic Press, New York, 1979), Vol. 34.

[45] A. J. Minnich, J. A. Johnson, A. J. Schmidt, K. Esfarjani, M. S. Dresselhaus, K. A. Nelson, and G. Chen, Phys. Rev. Lett. 107, 095901 (2011).

[46] J. A. Johnson, A. A. Maznev, J. Cuffe, J. K. Eliason, A. J. Minnich, T. Kehoe, C. M. S. Torres, G. Chen, and K. A. Nelson, Phys. Rev. Lett. 110, 025901 (2013).

[47] K. T. Regner, D. P. Sellan, Z. Su, C. H. Amon, A. J. H McGaughey, and J. A. Malen, Nat. Commun. 4, 1640 (2013).

[48] V. Popescu and A. Zunger, Phys. Rev. B 85, 085201 (2012).

[49] P. V. C. Medeiros, S. Stafström, and J. Björk, Phys. Rev. B 89, 041407 (2014).

[50] G. Antonius, S. Poncé, P. Boulanger, M. Côté, and X. Gonze, Phys. Rev. Lett. 112, 215501 (2014). 